\title{
Droplet Interfaced Parallel and Quantitative Microfluidic-Based Separations
}

\author{
${ }_{3}$ Sammer-ul Hassan, ${ }^{\dagger}$ Hywel Morgan, ${ }^{\ddagger}, \S$ Xunli Zhang, ${ }^{\dagger, \S}$ and Xize Niu* ${ }^{* \dagger, \S}$ \\ $4{ }^{\dagger}$ Faculty of Engineering and the Environment, University of Southampton, Southampton, U.K. SO17 1BJ \\ 5 "Faculty of Physical Sciences and Engineering, University of Southampton, Southampton, U.K. SO17 1BJ \\ ${ }_{6}{ }^{\S}$ Institute for Life Sciences, University of Southampton, Southampton, U.K. SO17 1BJ
}

\section{S Supporting Information}

8 ABSTRACT: High-throughput, quantitative, and rapid microfluidicbased separations has been a long-sought goal for applications in proteomics, genomics, biomarker discovery, and clinical diagnostics. Using droplet-interfaced microchip electrophoresis (MCE) techniques, we have developed a novel parallel MCE platform, based on the concept of combining the Slipchip principle with a newly developed "Gelchip". The platform consists of two plastic plates, with droplet wells on one plate and separation channels with preloaded/cured gel in the other. A single relative movement of one plate enables generation and then loading of multiple sample droplets in parallel into the separation channels, allowing electrophoretic separation of biomolecules in the droplets in parallel and with high-throughput. As proof of concept, we demonstrated the

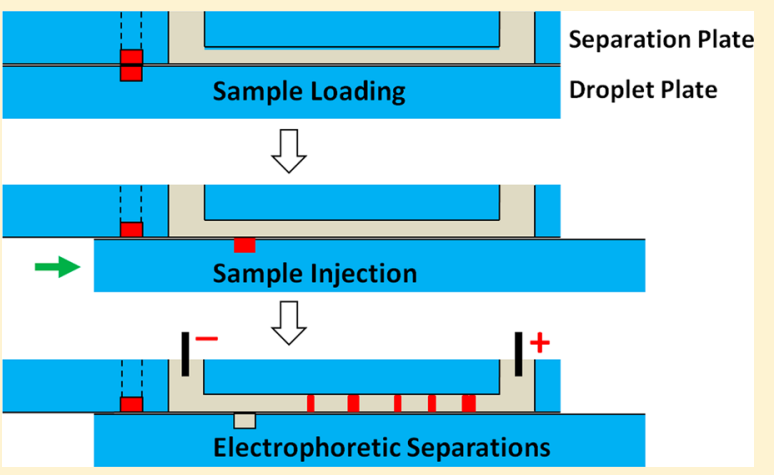
separation of 30 sub-nL sample droplets containing fluorescent dyes or DNA fragments.

${ }_{22}^{22}$ lectrophoresis in all its embodiments is a powerful analytical technique which has been applied to resolve 24 complex mixtures containing DNA, proteins, and other 25 chemical or biological species. ${ }^{1-4}$ The development of 26 microfabrication techniques has led to further miniaturization 27 of electrophoresis known as microchip electrophoresis (MCE), 28 which offers particular advantages including ultrasmall volume 29 sample consumption, integration with other "lab-chip" 30 processes or functions such as extraction, purification, washing, 31 mixing, and sample concentration. ${ }^{5,6}$ As a result, MCE has been 32 used in a variety of applications, e.g., to analyze biomolecules in 33 blood, $^{7}$ saliva, ${ }^{8}$ tear, ${ }^{9}$ dialysate, ${ }^{10}$ and islets. ${ }^{11}$

34 The majority of MCE and capillary electrophoresis (CE) 35 technologies have used one of the two common sample 36 injection methods, i.e., electrokinetic or hydrostatic injection. In 37 the former, the sample injection may introduce bias as different 38 analytes have different electrophoretic mobilities; ${ }^{12,13}$ therefore, 39 the injected sample may not reflect the concentration and 40 composition of the original sample. ${ }^{4}$ Hydrostatic sample 41 injection technique has many difficulties, e.g., in controlling 42 the flow in the small microchannels, and has limited 43 throughput. $^{14}$ To analyze samples in parallel, microfluidic 44 chips have been fabricated that consist of arrays of micro45 channels. ${ }^{12,15-19}$ However, these devices use the same sample 46 injection methods listed above. Pan et al. has recently shown an 47 elegant method of parallel separation in free-standing gel strips 48 with 96 wells, and this could lead to high throughput and 49 quantitative analysis with a low running cost. ${ }^{20}$
Droplet-based microfluidics has immerged as a powerful tool, 50 and the technique can encapsulate biological samples in 51 discrete droplets, enabling manipulation and analysis in a 52 high throughput format. ${ }^{21-24}$ Subnanoliter sample droplets can 53 be generated in a microfluidic chip or collected from a 54 bioreactor, an upstream separation column, or even from a 55 tissue environment. ${ }^{25-27}$ These discrete sample droplets can be 56 further analyzed by electrophoresis by injecting them into a 57 separation channel. ${ }^{28,29}$ Such droplet-interfaced systems have 58 been shown to be effective for sample injection and offer many 59 other advantages including ultrasmall volume consumption, no 60 sample waste, quantitative analysis without bias, simple device 61 setup, and no electric field switching. Interestingly such systems 62 also reintroduce $\mathrm{CE}$ as a powerful analytical tool to resolve 63 complex mixtures within microdroplets. ${ }^{10,29}$ However, in these 64 droplet-interfaced separations, sample droplets are mostly 65 analyzed in serial that limits throughput. Jian ${ }^{30}$ has addressed 66 this shortcoming by expanding an original single separation 67 channel to three, but further multiplexing requires complicated 68 droplet manipulation, which may not be feasible.

The Slipchip, first developed by Ismagilov's group, ${ }^{31}$ is an 70 effective new method for parallel droplet operations. It is a 71 simple device consisting of two plates with small wells 72 fabricated in each. The wells can be filled with different 73 reagents and upon sliding one layer relative to the other, 74

Received: December 17, 2014

Accepted: March 16, 2015 

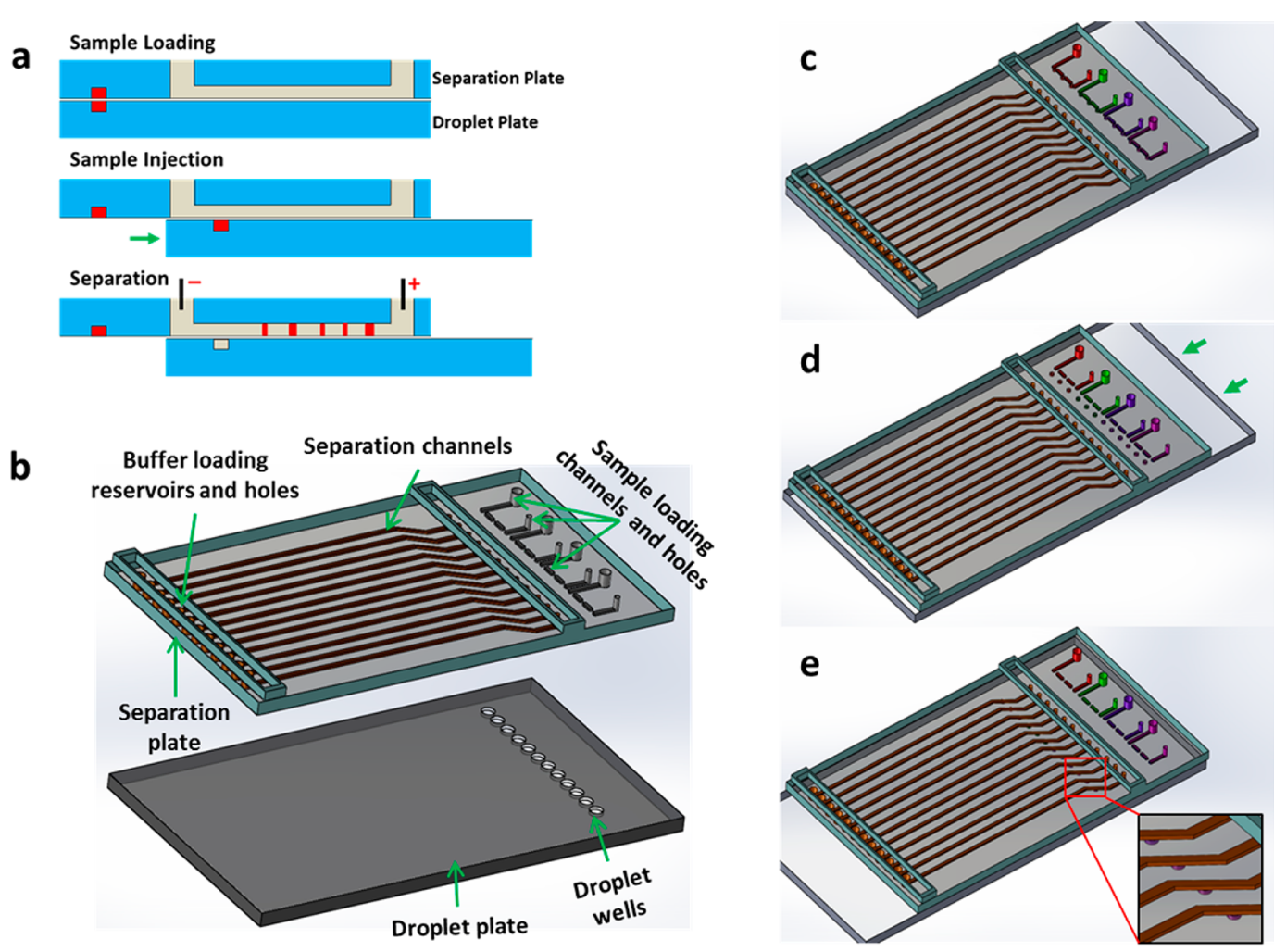

Figure 1.3D Schematic showing the working principle of the device (dimensions are not drawn to scale). (a) Schematic view of droplet generation, injection, and sample separation. The arrow indicates the movement direction of the droplet plate. (b) Schematic of the separation plate and droplet plate. (c) Initial position of chips after assembly and loading samples. (d) Slipping bottom layer to generate droplets (arrows show the movement direction of the droplet plate). (e) Injection of droplets into the separation channels.

75 various operations can be implemented such as generation of 76 sample droplets or fusion of droplets to initiate chemical 77 reactions. $\mathrm{We}^{32}$ and later Shujun et al. ${ }^{33}$ demonstrated that this 78 Slipchip format has the potential to be used for separation 79 science, e.g., for segmenting separated samples after isoelectric 80 focusing (IEF) into microdroplets to avoid any sample remixing 81 during postseparation sample collection.

82 Herein, we combine the concepts of the Slipchip and MCE 83 and develop a novel device that can achieve parallel droplet 84 interfaced separations, by loading droplets into separation gel/ 85 medium with the "slipping" function. With this sample loading 86 method, the entire volume of the sample droplets can be 87 separated. Therefore, it offers the ability to quantify 88 biomolecules in sample droplets.

\section{$89 \square$ EXPERIMENTAL SECTION}

90 Materials. Fluorescein 5(6)-isothiocyanate (FITC), fluo91 rescein, 5-carboxyfluorescein, eosin Y, tris borate buffer (TBE), 92 and agarose powder were obtained from Sigma-Aldrich 93 (Dorset, U.K). Poly(ethylene oxide) (PEO, $500 \mathrm{kDa}$ ) was 94 purchased from Avocado Research Chemicals Ltd. (Lancashire, 95 U.K.). Solutions of $30 \%(\mathrm{w} / \mathrm{v})$ acrylamide/bis(acrylamide), 96 cross-linker (TEMED), initiator (ammonium persulfate), and 97 sodium dodecyl sulfate (SDS) were also purchased from Sigma98 Aldrich (Dorset, U.K). DNA ladder (Mapmarker FAM labeled) 99 was purchased from BioVentures Inc. (Murfreesboro).

100 Sample Preparation. Commercially available reagents 101 were bought and used without further purification. Fluorescein 102 5(6)-isothiocyanate (FITC), fluorescein, 5-carboxyfluorescein, 103 and eosin $\mathrm{Y}$ were dissolved in $0.1 \times \mathrm{TBE}$ at a stock 104 concentrations of $300 \mu \mathrm{M}$. DNA samples were prepared by mixing standard Mapmarker ladder with formamide at equal 105 volumes and diluted with $1 \times \mathrm{TBE}$ to achieve $10 \times$ diluted 106 standard sample. The sample mixture was predenatured at 95107 ${ }^{\circ} \mathrm{C}$ for $2 \mathrm{~min}$ and snap-cooled on ice prior to loading to the 108 sample channels.

Microchip Fabrication and Preparation. The micro- 110 fluidic chips used for all experiments were fabricated by a 111 precise micromilling in poly(methyl methacrylate) (PMMA) 112 sheets using an LPKF micromilling machine (ProtoMat-S100). 113 The separation channels $(150 \mu \mathrm{m} \times 200 \mu \mathrm{m}$ width $\times$ depth, 7114 $\mathrm{cm}$ long), via holes (300 $\mu \mathrm{m}$ diameter), cathode and anode 115 reservoirs were milled on the top plate, while microwells for 116 sample droplets $(150 \mu \mathrm{m} \times 200 \mu \mathrm{m} \times 200 \mu \mathrm{m}$ width $\times$ length 117 $\times$ depth) were milled on the bottom plate. The chip surface 118 was rendered smooth using chemical reflow. ${ }^{34}$ Briefly, a small 119 container was filled with chloroform and the microchip was 120 placed on top of the container with all the channels exposed to 121 chloroform vapor. The distance between the microchip and the 122 chloroform layer was kept at $5 \mathrm{~mm}$ and an exposure time of 3- 123 4 min was found to be suitable for reflow of the PMMA surface. 124 A longer exposure time of more than $4 \mathrm{~min}$ was found to 125 damage the channels and cracks appeared on the surface. 126 Chloroform is a hazardous material and the vapors can cause 127 acute toxicity, irritation, or carcinogenicity. Therefore, the 128 reflow process was performed in a fumehood and personal 129 protective equipment were used. Both of the MCE chips were 130 treated with Duxback (Duxback Ltd.) and heated at $65{ }^{\circ} \mathrm{C}$ for 131 $10 \mathrm{~min}$ in an oven to evaporate the solvent making the PMMA 132 surface hydrophobic. Before use, 30-50 $\mu \mathrm{L}$ of fluorinated oil 133 (FC-40) was spread over the chip to wet the surface thus 134 preventing leakage of sample from the wells during slipping. 135 
a

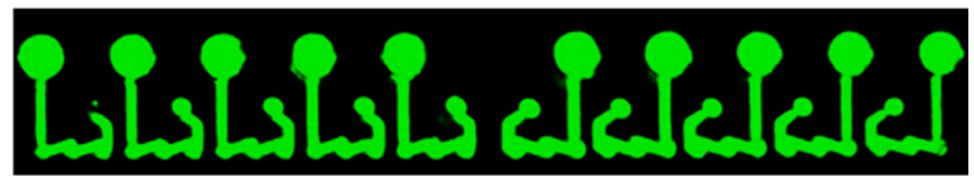

b
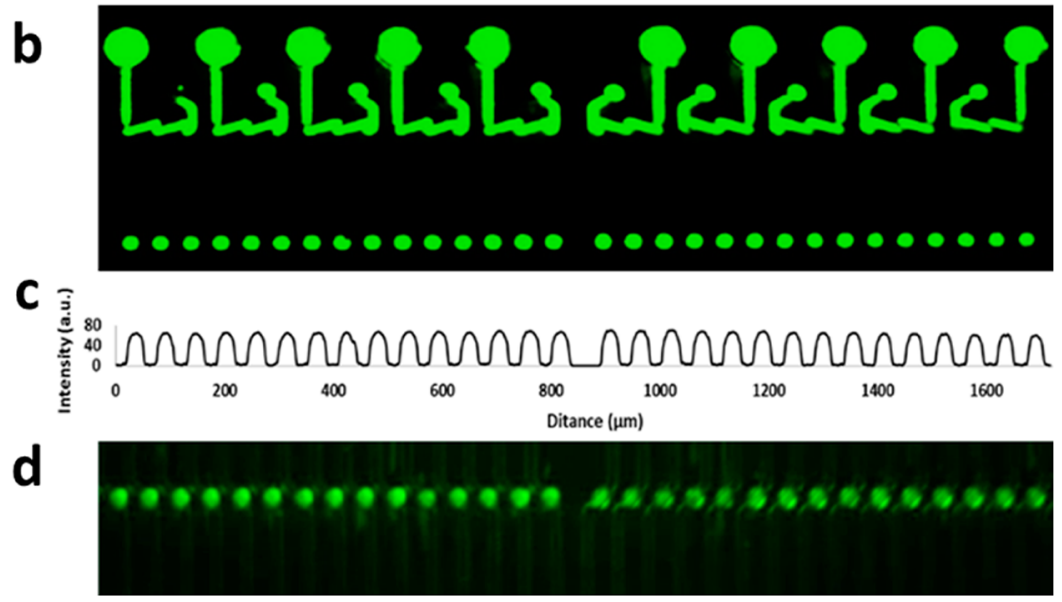

Figure 2. Droplet generation and injection. (a) Fluorescein filled in the sample loading channels. (b) Droplets generated after slipping the chip. (c) Fluorescence intensity profile of the droplets. (d) Droplets injected into separation channels.

136 The sample and buffer were loaded into the microchip by a 137 pipet.

138 Microchip Design and Operation. Figure 1a shows the 139 schematic diagram of the chip design and operation. The chip 140 consists of separation channels, sample loading channels, buffer 141 reservoirs together with holes on the top plate and droplet wells 142 on the bottom plate. Henceforth, the top plate is referred to as 143 the separation plate and the bottom plate as the droplet plate. 144 After fabrication with PMMA material, the plate surfaces 145 were smoothed by reflowing with chloroform and coating with 146 Duxback. This produced surfaces with excellent optical clarity 147 and hydrophobicity (see Figure S1 in the Supporting 148 Information). To facilitate "nonmicrofluidic users", we have 149 also developed protocols that precure separation gels (e.g., 150 agarose, polyacrylamide) in open channels forming a "Gelchip" 151 that can be prepared in batch and used off-the-shelf. The 152 separation plate was joined to the droplet plate in such a way 153 that the sample loading channels were connected with droplet 154 wells forming zigzag channels as shown in Figure 1a.

155 To seal the microchannels and minimize sample sticking on 156 the surface or any leakage, fluorinated oil (FC-40) was added at 157 the interface, especially covering the areas between the droplet 158 generation and the sample loading channels. The oil also 159 lubricates the two plates and minimizes surface friction during 160 movement of the plates. The two chip halves were clamped 161 together using magnets on opposite sides to ensure a tight 162 contact of the two plates. ${ }^{32}$ After assembly, the sample mixture 163 was loaded into the sample loading channels via inlet holes $(0.8$ $164 \mathrm{~mm}$ diameter) that were fitted with the end of a pipet tip. The 165 separation buffer (TBE/Tris-Ches) was then loaded to the two 166 buffer reservoirs connecting to both ends of the separation 167 channels. The $0.5 \mathrm{~mm}$ diameter platinum wire electrodes were 168 placed in each reservoir to provide a uniform electric field to all 169 of the parallel channels. Noted in certain separation modes 170 when liquid sieving matrixes are required, such as poly(ethylene 171 oxide) (PEO) solution for capillary gel electrophoresis (CGE) 172 or other capillary zone electrophoresis (CZE) buffers, the 173 separation channels were left empty during chip assembly. 174 Separation matrixes were then added to the channels before sample loading, by gentle liquid pumping to the inlet holes with 175 a peristaltic pump at a flow rate of $3 \mu \mathrm{L} / \mathrm{min}$. The droplet plate 176 was then moved by a micrometer connected to the droplet 177 plate from its initial position; thereby generating droplets in 178 each of the wells (Figure 1d,e). During this process, sample 179 droplets were first generated from the sample channels and 180 further moved to overlap with the separation channels. Finally a 181 dc electric field was applied across the reservoirs and migrating 182 the sample molecule toward the opposite charge end 183 performing electrophoresis separations.

Droplet Generation and Injection. Droplet generation 185 and injection were initially calibrated by loading fluorescein dye 186 solution into the sample loading (zigzag) channels connected 187 to the droplet wells (Figure 2a). After slipping the chip, each $188 \mathrm{f2}$ sample produced 3 repeat droplets, as shown in Figure 2b. 189 Fluorescence intensity profiles of the generated droplets gives 190 information on the reproducibility of the droplet area (Figure 191 2c). The percent relative standard deviation (\% RSD) for all of 192 the 30 droplets in more than 3 runs was $<3 \%$. In Figure 2d, 193 droplets were moved to be in contact with the separation 194 channels. There was no surfactant added into the oil; therefore, 195 the aqueous droplet immediately merged with the gel and 196 sample molecule started to diffuse into the separation channels. 197 Confocal imaging taken $30 \mathrm{~s}$ after droplet merging showed that 198 the fluorescent molecules had already diffused into the 199 separation gel (Figure S6 in the Supporting Information). 200 Droplet interfacing is a new approach to sample loading, relying 201 on segmentation, and loading of sample droplets; therefore, 202 appropriate oil is needed to prevent unwanted droplet breakup, 203 sample leakage, or surface contamination. The FC-40 oil 204 membrane trapped in between the two plates kept the aqueous 205 sample droplets inside the droplet wells while it was moved 206 toward the separation channels. However, the detergent SDS 207 was found to destabilize the oil-water interface (FC-40 oil and 208 TBE buffer combination) causing severe sample loss into the 209 interface of the two plates; therefore, we do not recommend 210 adding SDS into the sample for this method. 


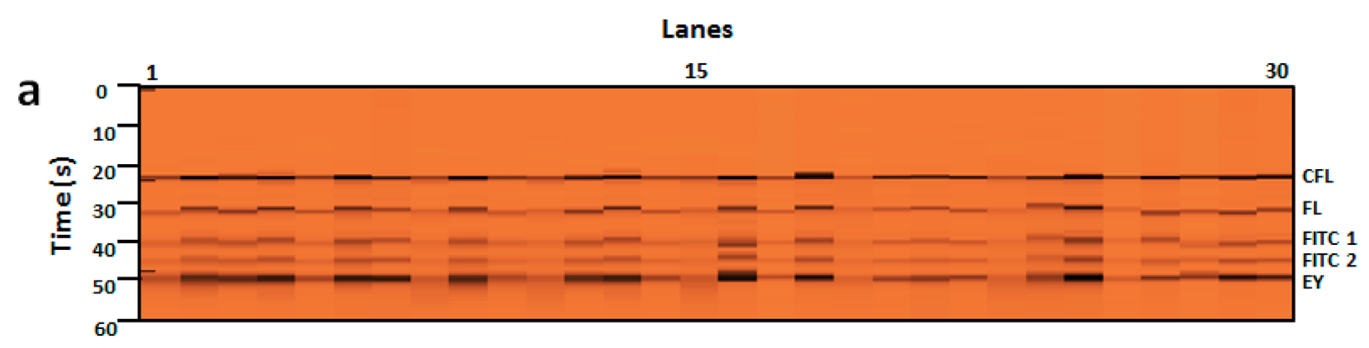

b
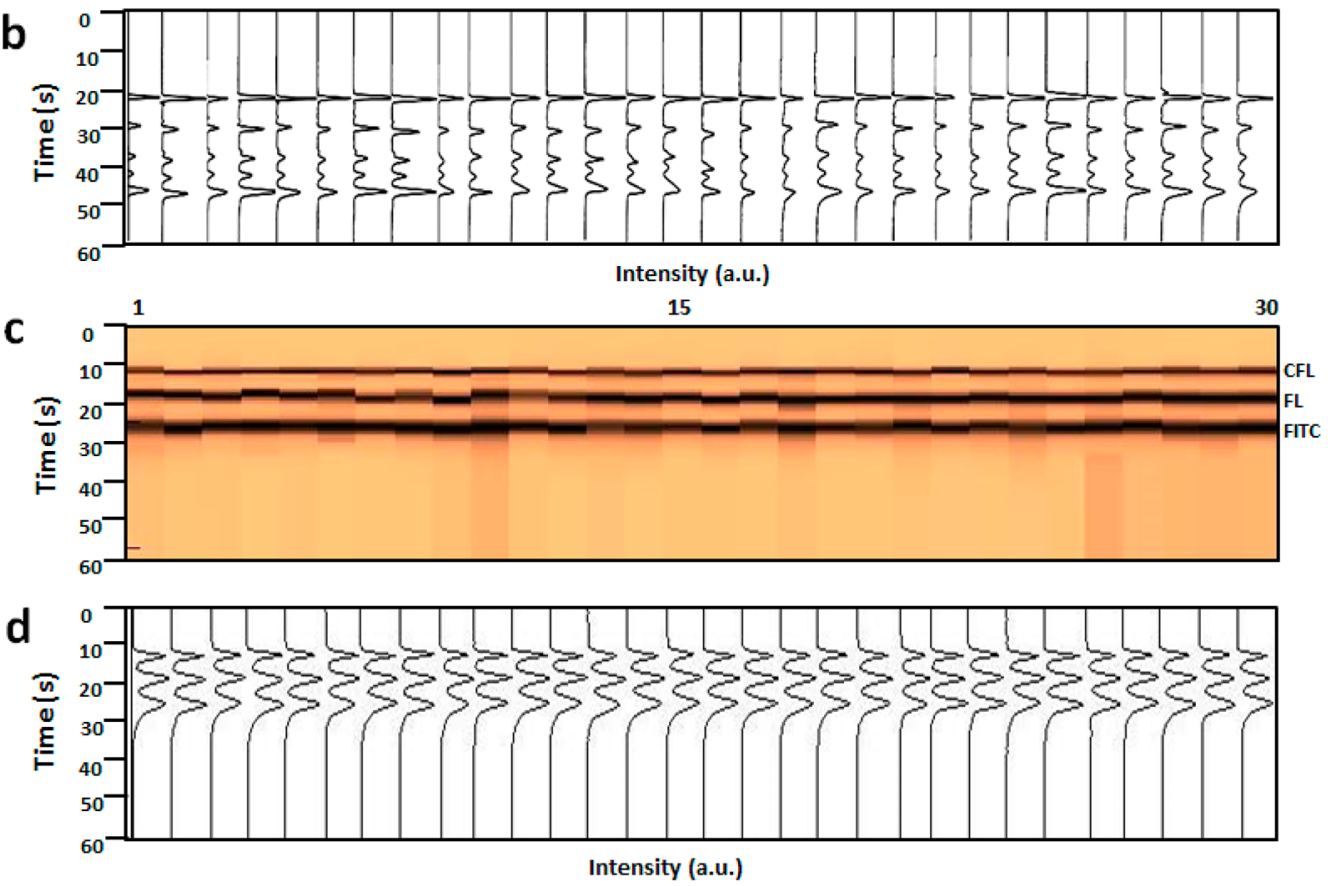

e

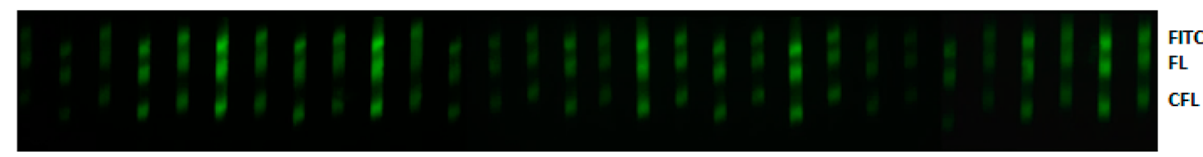

Figure 3. Separation of fluorescent dyes in PEO gel (a, b) and agarose gel (c, d, e). (a) Pseudo gel plot from a PEO gel separation for sample mixture (Eosin Y, FITC 1, FITC 2, fluorescein, and 5-carboxyFL). (b) Corresponding electropherograms. Field strength, $90 \mathrm{~V} / \mathrm{cm}$; detection point, $3.5 \mathrm{~cm}$; separation medium, 1.5\% PEO gel. (c) Pseudo gel plot from an agarose gel separation for sample mixture (FITC, fluorescein, and 5-carboxyFL). (d) Corresponding electropherograms. (e) Microscope snapshot of the separated bands. Field strength, $80 \mathrm{~V} / \mathrm{cm}$; detection point, $6 \mathrm{~mm}$; separation medium, $2 \%$ agarose gel.

\section{RESULTS AND DISCUSSION}

213 Separation reproducibility was determined by separating 214 fluorescent dyes in the microchannels. In this experiment, all 215 the sample droplets contained the same sample mixture. FITC, 216 fluorescein, eosin $\mathrm{Y}$, and 5-carboxyfluorescein at concentrations 217 of $25,10,144$, and $13.5 \mu \mathrm{M}$, respectively, were prepared in $0.1 \times$ $218 \mathrm{TBE}$ buffer and separated using $1.5 \%$ PEO gel ( $500 \mathrm{kDa})$. The 219 dyes were negatively charged at $\mathrm{pH} 8.4$ and migrated toward 220 the anode when an electric field $(90 \mathrm{~V} / \mathrm{cm})$ was applied. The 221 bands of separated dyes were detected at a distance of $3.5 \mathrm{~cm}$ 222 from the sample injection point. The pseudo gel plot and 223 representative electropherograms were drawn in Figure 3a,b, 224 from the fluorescent intensity data collected from recorded 225 videos. The electropherogram was realigned according to the 226 peaks from 5-carboxyfluorescein as an internal standard. It is 227 clearly seen that baseline separation was achieved and the 228 separation was completed in $60 \mathrm{~s}$. The apparent number of 229 theoretical plates, an indicator of maximum separation 230 efficiency of a separation column/channel is given by $N=$ ${ }_{231} 5.54\left[t_{\mathrm{r}} / W_{t_{1 / 2}}\right]^{2}$, where $W_{t_{1 / 2}}$ is the width of the peak at half of the height (expressed in terms of time) and $t_{\mathrm{r}}$ is the retention time 232 of the separated molecule, both were measured from recorded 233 videos using a homemade Matlab (Mathworks) program. 234 Theoretical plates were calculated to be 7560 at a distance of 235 $3.5 \mathrm{~cm}$. This value is 1 order of magnitude less than for glass 236 chip-based separations. ${ }^{35}$ This lower number of theoretical 237 plates can be attributed to three main factors. First, there was 238 molecular diffusion at the injection point and in the separation 239 channels. The diffusion was more obvious for small fluorescent 240 molecules than larger biomolecules (as shown in the later DNA 241 separation with higher plate numbers); second, separation 242 conditions such as buffer/gel concentrations, surface coating, 243 and electric field strength have not been optimized in this 244 study; and third, the droplet volume (800 pL) used here was 245 larger compared to the $10 \mathrm{~ms}$ injection time in a cross-piece 246 injections. $^{35}$ The separation reproducibility was also calibrated 247 in the precasted agarose gel ( $2 \%$ agarose), as shown in Figure 248 $3 \mathrm{c}, \mathrm{d}$. The theoretical plates were calculated to be 1890 at a 249 distance of $6 \mathrm{~mm}$. Supplementary Movie 3 in the Supporting 250 
251 Information provides a short recording for the separation of 252 these dyes in 15 channels filled with agarose gel.

253 We further determined the effect of droplet sizes on the 254 separation efficiency. Four different droplet wells were 255 fabricated with different well depths to generate droplets with 256 volumes of $320,800,1280$, and $1750 \mathrm{pL}$ as shown in Figure 257 S4a,b in the Supporting Information. FITC and fluorescein 258 were separated within these four different droplet sizes (Figure $259 \mathrm{~S} 4 \mathrm{c}, \mathrm{d}$ in the Supporting Information) and separation resolution 260 (SR) was determined. It was found that the SR decreases with 261 the increasing droplet volume. The bands were highly resolved 262 for the smallest droplet volume (Figure S5e in the Supporting 263 Information). However, there are slight deviations from a linear 264 fit which could be due to the differences in droplet size, 265 variations in electric field strength in different channels, and 266 wettability of the channels which affects aqueous droplet 267 merging. The theoretical plates achieved by this separation are 2682220 (corresponding to the smallest droplet) to 1480 (the 269 largest droplet) at a distance of $8 \mathrm{~mm}$.

270 This method of droplet-interfaced separation allows for 271 whole sample injection from the droplets to the separation 272 channels without any sample waste. Therefore, quantitative 273 analysis of analytes within the sample mixture can be achieved. 274 For each sample mixture, the chip produces multiple sample 275 droplets (three in this setup). The separation results can be 276 compared to provide a standard derivation, as is generally 277 required in a biochemical analysis. Since these sample repeats 278 are analyzed in parallel, no extra separation time is required in 279 our system. To validate this method, mixtures of samples were 280 prepared with fixed concentrations of 5-carboxyluorescein (9 $281 \mu \mathrm{M})$ and fluorescein $(50 \mu \mathrm{M})$ and varying the FITC 282 concentration from 0 to 50,100, 150, 200, and $250 \mu \mathrm{M}$. 283 Each sample was injected into one sample channel to produce 284 three droplet copies, which were then separated in correspond285 ing channels and the results are shown in Figure 4. A program 286 written in Matlab was used to extract the peak areas, which 287 were further normalized using the peak area of 5-carboxy288 fluorescein as an internal standard. The change in peak areas 289 has a linear correspondence (3.6\% RSD) to the original sample 290 concentrations as illustrated in Figure 4c.

291 DNA sizing and protein separation are important applica292 tions of gel electrophoresis in biochemistry, forensics, and 293 immunoassays. Here DNA ladders from 50, 100, 150, 200, 300, 294 400, 500, and $600 \mathrm{bp}$ were separated to assess the performance 295 of the device with PEO gel, which is a well-studied sieving 296 matrix for separating DNA fragments ranging from $25 \mathrm{bp}$ to 297 over $2000 \mathrm{bp} .{ }^{3,36}$ With the gel loading methods described in the 298 Experimental Section, different PEO (500 kDa) concentrations 299 ranging from 0.5 to $3 \%$ were successfully loaded to separation 300 channels and the DNA ladder was best separated in $2.5 \%$ PEO 301 gel in the device. Figure 5 shows the detection at a distance of $30213 \mathrm{~mm}$ from the point of injection, and the separation was 303 completed within $120 \mathrm{~s}$. The number of theoretical plates were 304 calculated to be 79800 , comparable with the other microchip 305 based DNA separations using microchips, ${ }^{37}$ which is 1 order of 306 magnitude higher than previously achieved theoretical plates 307 using our droplet interfaced microfluidic chips. ${ }^{29}$

\section{CONCLUSION AND DISCUSSION}

309 In this paper, we have developed a novel droplet-interfaced 310 microchip electrophoresis device that provides parallel and 311 quantitative separations of analytes from subnano liter droplets. 312 The chip contains precured agarose or polyacrylamide gel,
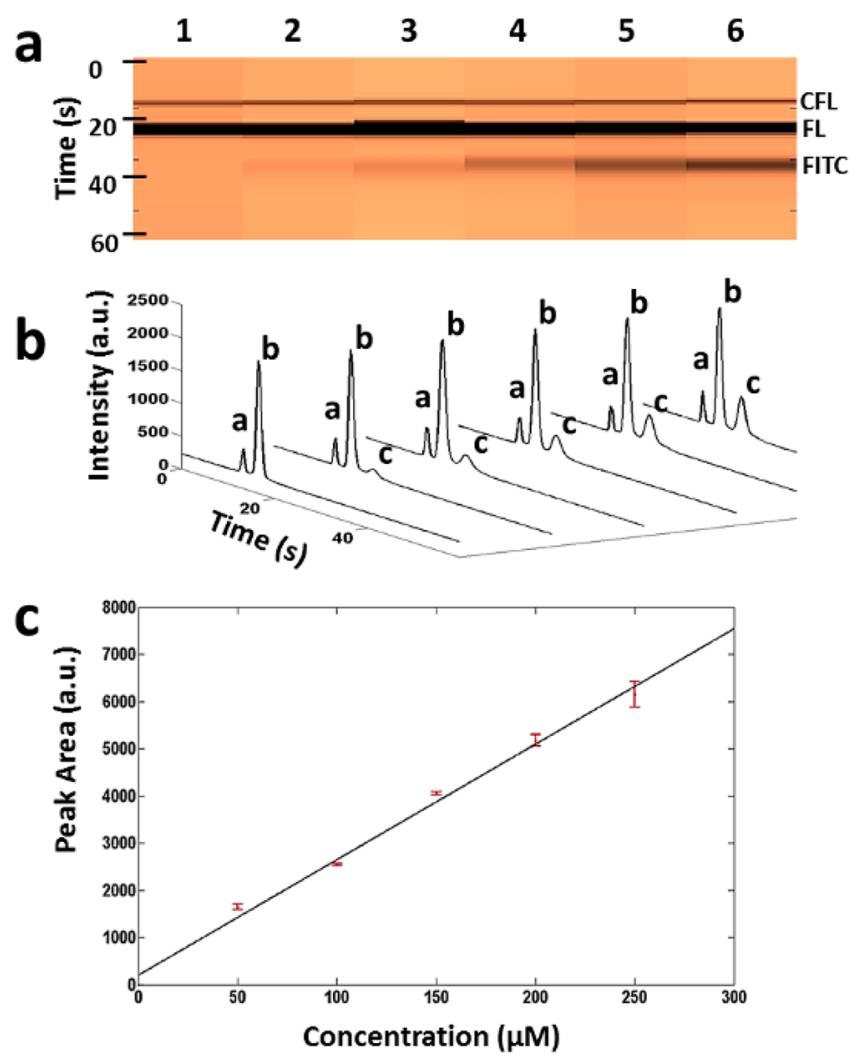

Figure 4. Quantitative analysis of sample droplets. (a) Pseudo gel plot. (b) Electropherograms. (c) Standard curve for peak area vs concentrations of FITC. FITC concentrations from left to right are $0,50,100,150,200,250 \mu \mathrm{M}$. Field strength, $80 \mathrm{~V} / \mathrm{cm}$; detection point, $8 \mathrm{~mm}$; separation medium, $2 \%$ agarose gel.

hence the name "Gelchip", and can be used off-the-shelf. A 313 liquid separation medium (e.g., PEO) can also be loaded into 314 the channels in situ; therefore, the device supports a wide range 315 of separation methods. Although in our initial study, each 316 channel was loaded with a homogeneous gel, advanced 317 separation abilities could be added using gels with gradients ${ }^{38} 318$ or forming preconcentration zones within the channels ${ }^{39}$ or 319 online labeling by curing derivatization dyes into the gel. ${ }^{40} \quad 320$

In the prototype, sample droplets were generated by one slip 321 of the chip and the sample breaks up in the zigzag channels to 322 form droplets. Droplets could also be pregenerated by the other 323 droplet generation or collection methods or devices and 324 trapped in these zigzag channels/wells for CE analysis. While 325 on-chip PCR and immunoassay functions have been demon- 326 strated for Slipchips, multiple step assays could be integrated 327 leading to a self-contained complex diagnostic device. After 328 separation, the Slipchip plates can be detached and the gel can 329 be used for other analytical methods or exposed to other 330 chemicals. This could facilitate postseparation staining and 331 destaining or MALDI MS that are under study. The device is 332 user-friendly and has the potential to be applied for DNA 333 sizing, peptide and protein separation, or immunoassays in a 334 high-throughput format using minute amounts of sample. With 335 future improvement in the detection capacity and sensitivity of 336 the system, the device has the potential to be applied for high- 337 resolution analysis of complex mixtures with hundreds of 338 droplets or for $2 \mathrm{D}$ separations of serum proteins, quantitative 339 immunoassays, or Western blot analyses. 

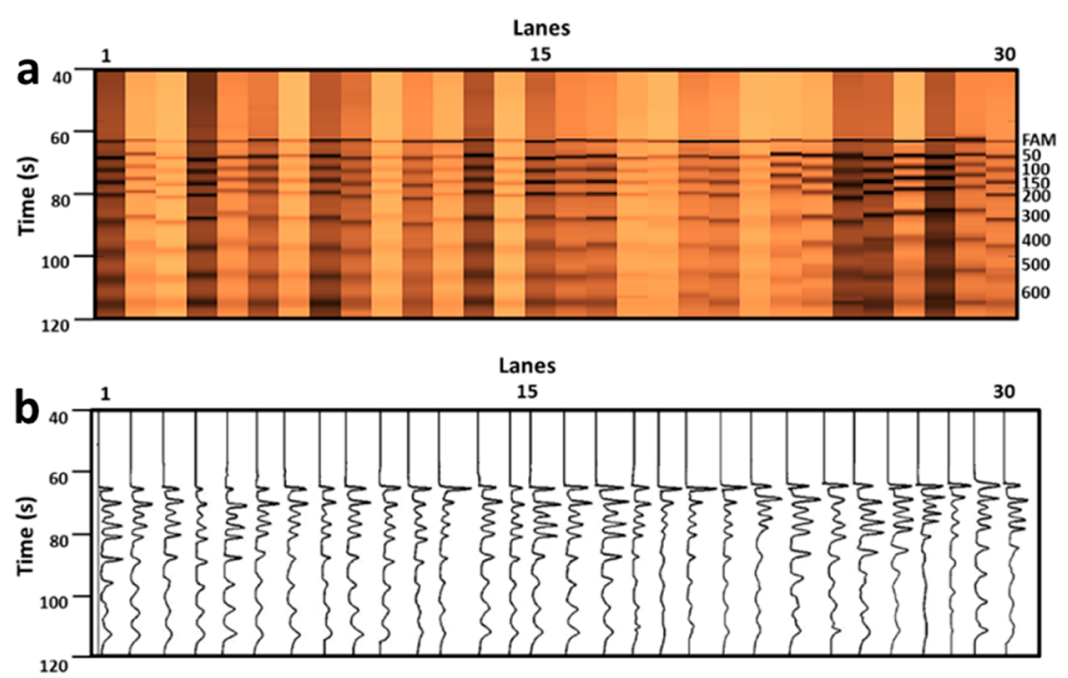

Figure 5. Separation of 50, 100, 150, 200, 300, 400, 500, and 600 bp DNA ladder (BioVentures Mapmarker FAM labeled), (a) pseudo gel plot of DNA fragments. (b) Electropherograms. Field strength, $100 \mathrm{~V} / \mathrm{cm}$; detection point, $1.3 \mathrm{~cm}$; separation medium, $2.5 \%$ PEO.

\section{ASSOCIATED CONTENT}

\section{S Supporting Information}

343 Fabricated chip plates, gel curing protocol, microchip 344 separation and data analysis, calibration of the relationship 345 between droplet volume and separation resolution, and 346 supplementary movies (3D movie of droplet injection, 347 fluorescein movie of droplet injection, and separation video). 348 This material is available free of charge via the Internet at 349 http://pubs.acs.org.

\section{AUTHOR INFORMATION}

\section{Corresponding Author}

352 *E-mail: x.niu@soton.ac.uk.

353 Notes

354 The authors declare no competing financial interest.

355

\section{ACKNOWLEDGMENTS}

356 This work was partially supported by the Engineering and 357 Physical Sciences Research Council UK (Grant EP/M012425/ 358 1), and we thank Mr. Junjun Lei for help on COMSOL 359 Simulation. H.M. would like to acknowledge the Royal Society 360 for funding.

\section{$361 \square$ REFERENCES}

362 (1) Ostergaard, J.; Jensen, H. Anal. Chem. 2009, 81, 8644-8648.

363 (2) Mosca, A.; Paleari, R.; Mosca, L.; Marcello, A.; Vercellati, C.; 364 Zanella, A. Clin. Biochem. 2009, 42, 1859.

365 (3) Pereira, F.; Hassard, S.; Hassard, J.; deMello, A. Electrophoresis $3662009,30,2100-2109$.

367 (4) Huang, X. H. C.; Quesada, M. A.; Mathies, R. A. Anal. Chem. 368 1992, 64, 2149-2154.

369 (5) Haeberle, S.; Zengerleab, R. Lab Chip 2007, 7, 1094-1110.

370 (6) Roddy, E. S.; Xu, H.; Ewing, A. G. Electrophoresis 2004, 229-42, 371229.

372 (7) Koutny, L. B.; Schmalzing, D.; Taylor, T. A.; Fuchs, M. Anal. 373 Chem. 1996, 68, 18-22.

374 (8) Herr, A. E.; Hatch, A. V.; Throckmorton, D. J.; Tran, H. M.; 375 Brennan, J. S.; Giannobile, W. V.; Singh, A. K. Proc. Natl. Acad. Sci. 376 U.S.A. 2007, 104, 5268-5273.

377 (9) Karns, K.; Herr, A. E. Anal. Chem. 2011, 83, 8115-8122.

378 (10) Wang, M.; Roman, G. T.; Perry, M. L.; Kennedy, R. T. Anal. 379 Chem. 2009, 81, 9072-9078.
(11) Dishinger, J. F.; Reid, K. R.; Kennedy, R. T. Anal. Chem. 2009, 380 81, 3119-3127. 381

(12) Harrison, D. J.; Manz, A.; Fan, Z. H.; Ludi, H.; Widmer, H. M. 382 Anal. Chem. 1992, 64, 1926-1932.

(13) Ermakov, S. V.; Jacobson, S. C.; Ramsey, J. M. Anal. Chem. 384 2000, 72, 3512-3517. 385

(14) Tabuchi, M.; Kuramitsu, Y.; Nakamura, K.; Baba, Y. Anal. Chem. 386 2003, 75, 3799-3805. 387 (15) Yang, S.; Liu, J.; Lee, C. S.; DeVoe, D. L. Lab Chip 2009, 9, 388 592-599.

(16) Emrich, C. A.; Medintz, I. L.; Chu, W. K.; Mathies, R. A. Anal. 390 Chem. 2007, 79, 7360-7366.

(17) Gaunt, T. R.; Hinks, L. J.; Rassoulian, H.; Day, I. N. M. Nucleic 392 Acids Res. 2003, 31 (9), e48-e48.

(18) Paegel, B. M.; Emrich, C. A.; Wedemayer, G. J. Scherer, J. R; 394 Mathies, R. A. Proc. Natl. Acad. Sci. U.S.A. 2002, 99, 574-579. 395

(19) Bousse, L.; Mouradian, S.; Minalla, A.; Yee, H.; Williams, K.; 396 Dubrow, R. Anal. Chem. 2001, 73, 1207-1212.

(20) Pan, Y.; Duncombe, T. A.; Kellenberger, C. A.; Hammond, M. 398 C.; Herr, A. E. Anal. Chem. 2014, 86, 10357-10364.

(21) Chiu, D. T. TrAC, Trends Anal. Chem. 2003, 22, 528-536.

(22) Niu, X.; de Mello, A. J. Biochem. Soc. Trans. 2012, 40, 615-623. 401

(23) Utada, A. S.; Lorenceau, E.; Link, D. R.; Kaplan, P. D.; Stone, H. 402 A.; Weitz, D. A. Science 2005, 308, 537-541. (24) Song, H.; Chen, D. L.; Ismagilov, R. F. Angew. Chem., Int. Ed 404 2006, 45, 7336-7356.

(25) Niu, X. Z.; Zhang, B.; Marszalek, R. T.; Ces, O.; Edel, J. B.; 406 Kluga, D. R.; deMello, A. J. Chem. Commun. 2009, 41, 6159-6161. 407

(26) Draper, M. C.; Niu, X. Z.; Cho, S.; Jarnes, D. I.; Edel, J. B. Anal. 408 Chem. 2012, 84, 5801-5808.

(27) Edgar, J. S.; Milne, G.; Zhao, Y. Q.; Pabbati, C. P.; Lim, D. S. 410 W.; Chiu, D. T. Angew. Chem., Int. Ed 2009, 48, 2719-2722. 411

(28) Edgar, J. S.; Pabbati, C. P.; Lorenz, R. M.; He, M. Y.; Fiorini, G. 412 S.; Chiu, D. T. Anal. Chem. 2006, 78, 6948-6954. 413

(29) Niu, X.; Pereira, F.; Edel, J. B.; de Mello, A. J. Anal. Chem. 2013, 414 $85,8654-8660$.

(30) Pei, J.; Nie, J.; Kennedy, R. T. Anal. Chem. 2010, 82, 9261- 416 9267.

(31) Du, W.; Li, L.; Nichols, K. P.; Ismagilov, R. F. Lab Chip 2009, 9, 418 2286-2292.

(32) Zhao, Y.; Pereira, F.; de Mello, A. J.; Morgan, H.; Niu, X. Lab 420 Chip 2014, 14, 555-561.

(33) Wang, S.; Chen, S.; Wang, J.; Xu, P.; Luo, Y.; Nie, Z.; Du, W. 422 Electrophoresis 2014, 35, 2528-2533.

(34) Ogilvie, I. R. G.; Sieben, V. J.; Floquet, C. F. A.; Zmijan, R.; 424 Mowlem, M. C.; Morgan, H. J. Micromech. Microeng. 2010, 20, 065016. 425 
426 (35) Roman, G. T.; Wang, M.; Shultz, K. N.; Jennings, C.; Kennedy, 427 R. T. Anal. Chem. 2008, 80, 8231-8238.

428 (36) Tseng, W. L.; Chang, H. T. Electrophoresis 2001, 22, 763.

429 (37) Kang, C.; Back, S. K.; Song, I.; Choi, B.; Chang, J.; Cho, K.; 430 Kim, Y. Bull. Korean Chem. Soc. 2006, 27, 519-523.

431 (38) Hou, C.; Herr, A. E. Anal. Chem. 2010, 82, 3343-3351.

432 (39) Huang, H.; Xu, F.; Dai, Z. Electrophoresis 2005, 26, 2254-2260.

433 (40) Jin, L. J.; Giordano, B. C.; Landers, J. P. Anal. Chem. 2001, 73, 434 4994-4999. 